\title{
ON THE EXPECTED MAXIMUM DEGREE OF GABRIEL AND YAO GRAPHS
}

\author{
LUC DEVROYE, ${ }^{*}$ McGill University \\ JOACHIM GUDMUNDSSON, ${ }^{* *}$ NICTA \\ PAT MORIN, ${ }^{* * *}$ Carleton University
}

\begin{abstract}
Motivated by applications of Gabriel graphs and Yao graphs in wireless ad-hoc networks, we show that the maximum degree of a random Gabriel graph or Yao graph defined on $n$ points drawn uniformly at random from a unit square grows as $\Theta(\log n / \log \log n)$ in probability.
\end{abstract}

Keywords: Random geometric graph; Gabriel graph; Yao graph; maximum degree

2000 Mathematics Subject Classification: Primary 60D05

Secondary 68U05; 52C99

\section{Introduction}

Wireless ad-hoc networks consist of computers (or sensors) capable of communicating wirelessly with each other without any centralized information, infrastructure, or organization. A common mathematical model of such networks is the unit disk graph, in which the nodes consist of $n$ points in $\mathbb{R}^{2}$ and an edge exists between two nodes if and only if the distance between them is at most $r$. Depending on the value of $r$, which represents the transmission range of the wireless transmitters, the network can be anything ranging from a set of isolated vertices to the complete graph. The unit disk graph is also the basic model of continuum percolation theory [14].

The lack of centralized management and organization that occurs in ad-hoc networks means that individual nodes in the network typically have only local information about the nodes that they can communicate directly with. This makes even basic tasks, such as routeing, highly nontrivial because the combination of complete lack of organization and the unit disk graph topology is too unwieldy.

One approach to taming ad-hoc networks has been to compute the intersection of the unit disk graph with some 'nice' proximity graph, where the intersection of two graphs, $G_{1}=\left(V, E_{1}\right)$ and $G_{2}=\left(V, E_{2}\right)$, is the graph $\left(V, E_{1} \cap E_{2}\right)$. If the right proximity graph is chosen, the resulting graph will remain connected (if the original unit disk graph is connected) and will inherit some of the nice properties of the proximity graph. Ideally, the intersection can be computed locally, so that individual nodes can locally determine which of their incident edges belong to the intersection.

Received 15 June 2009; revision received 25 September 2009.

* Postal address: School of Computer Science, McGill University, 3480 University Street, Montreal, Québec, H3A 2A7, Canada.

** Postal address: NICTA, School of IT Building, J12 1 Cleveland Street, University of Sydney, NSW 2006, Australia.

*** Postal address: School of Computer Science, Carleton University, 1125 Colonel By Drive, Ottawa, Ontario, K1S 5B6, Canada. Email address: morin@scs.carleton.ca 
One such approach computes the intersection of the unit disk graph with the Gabriel graph [8]. The Gabriel graph contains an edge between two points $u$ and $v$ if and only if the disk whose diameter is $\|u v\|$ contains $u$ and $v$ on its boundary and no points other than $u$ and $v$ (see Figure 1). The Gabriel graph is planar and, therefore, has only a linear number of edges. Algorithms for routeing on planar graphs can be applied to the resulting graph or, more commonly, these algorithms can be used for recovery when routeing heuristics fail. A number of routeing algorithms and protocols have been proposed based on this strategy [2], [4], [11].

Another suggested approach uses the Yao graph [25]; see Figure 2. Let $p$ be a positive integer, let $\theta=2 \pi / p$, and let $u$ be a point in $\mathbb{R}^{2}$. The $i$-cone of $u$ is the set of all points $w \in \mathbb{R}^{2}$ such that the angle $L q u w \in[(i-1) \theta, i \theta)$, where $q=u+(1,0)$. The $\theta$-Yao graph contains an edge from $u$ to the nearest point in each of $u$ 's $i$-cones for $i=1, \ldots, p$. For any constant $p \geq 6$, the $\theta$-Yao graph has at most $p n$ edges and is a spanner; for any two vertices $u$ and $v$, the $\theta$-Yao graph contains a path whose Euclidean length is at most $t\|u v\|$, where $\|u v\|$ denotes the Euclidean distance between $u$ and $v$, and $t=1 /(1-2 \sin (\theta / 2))$ is called the stretch factor. When applied in the context of unit disk graphs, if there is a path of Euclidean length $\|u v\|_{U}$ in the original unit disk graph, then there is a path of length at most $t\|u v\|_{U}$ in the intersection

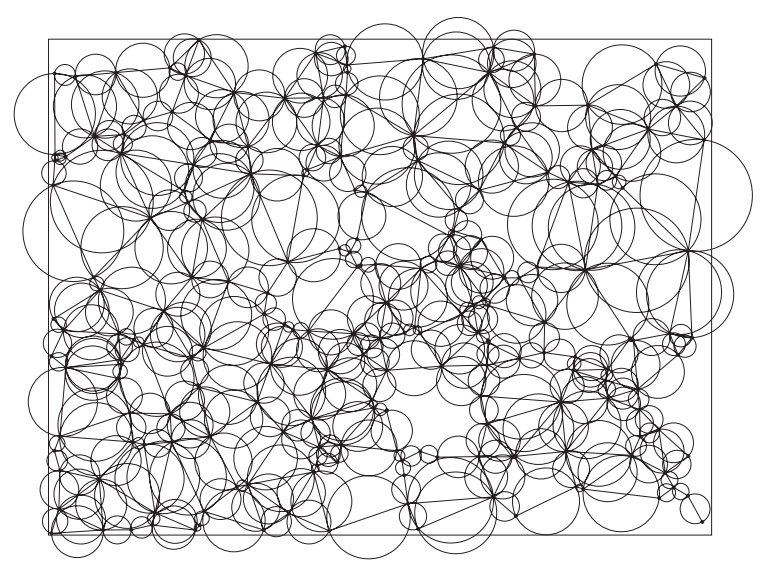

FIGURE 1: A point set with its Gabriel graph. No circle has any data point in its interior and every circle has an edge as its diameter.

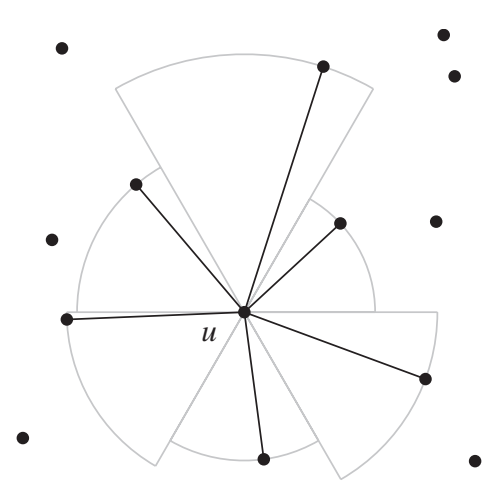

FIGURE 2: The edges defined by a node $u$ in a $(\pi / 3)$-Yao graph. 
of the unit disk graph and the $\theta$-Yao graph. Routeing strategies based on the Yao graph attempt to find power-efficient routeing paths [10], [12], [18], [24].

\subsection{New results}

Motivated by the above applications in wireless networks, in the current paper we study the Gabriel graph and Yao graph of $n$ points uniformly and independently distributed in a unit square. This distribution assumption can be used to approximately model the unorganized nature of ad-hoc networks and is commonly used in simulations of such networks [19]. Additionally, some types of sensor network, especially with military applications, are specifically designed to be deployed by randomly placing (scattering) them in the deployment area. This distribution assumption models these applications very well.

We show that the maximum degree of any node in a Gabriel graph or a Yao graph is concentrated at $\Theta(\log n /(\log \log n))$. (Throughout this paper $\log x$ denotes the natural logarithm of $x$.) More specifically, if $\Delta$ is the maximum degree of either graph then we show that there exist constants $a$ and $b$ such that

$$
\lim _{n \rightarrow \infty} \operatorname{Pr}\left\{\Delta \in\left[\frac{a \log n}{\log \log n}, \frac{b \log n}{\log \log n}\right]\right\}=1 .
$$

For Gabriel graphs, we show this for $(a, b)=\left(\frac{1}{12}, 1\right)$ and, for Yao graphs, we show it for $(a, b)=\left(\frac{1}{8}, 4\right)$. The maximum degree is particularly important in wireless networks, since the degree of a node directly impacts the amount of bookkeeping the node must do. With wireless nodes typically being battery operated and often memory and computationally constrained, the degree of a node should hopefully be as small as possible in order to minimize this bookkeeping.

\subsection{Related work}

The monograph by Penrose [16] presented a comprehensive treatment of the properties of unit disk graphs of points uniformly distributed in $[0,1]^{d}$, including connectivity, minimum and maximum degree, maximum clique size, and a number of other parameters.

A random Gabriel graph in this paper is a Gabriel graph for $n$ points drawn uniformly and at random from $[0,1]^{d}$. Its key properties were studied in great depth by Matula and Sokal [13]. For example, the expected number of edges grows as $2^{d-1} n$ [7], [13]. The length of an edge taken at random from all edges has expected value and standard deviation $\Theta\left(n^{-1 / d}\right)$ [7]. These properties hold also for many nonuniform distributions [7].

For a uniform Poisson process, introduced to avoid edge effects, Bern et al. [3] showed that the expected value of the maximum degree of a Delaunay triangulation grows as $\Theta(\log n / \log \log n)$. For that model, their proof also worked for Gabriel graphs. It is known that the Gabriel graph is a subgraph of the Delaunay triangulation (see [20]), so our upper bound on the maximum degree in a Gabriel graph would in fact follow without too much work from the cited paper. Our work on Gabriel graphs differs in three aspects.

1. We show convergence in probability. The fact that the expected maximum degree grows as $\Theta(\log n / \log \log n)$ does not imply that the probability of obtaining such large maximum degrees tends to 1 . We show that it does.

2. We deal with a fixed sample size model on a unit square, not the Poisson model on the entire plane.

3. Our proofs are different. 
The relative neighborhood graph is obtained by joining all pairs whose loon is empty, where the loon defined by a pair is the intersection of two spheres of equal radius, each having one point as its center and the other point on its surface (see [21]). As it is a subgraph of the Gabriel graph, our results imply that its maximum degree is $O(\log n / \log \log n)$ in probability. For a general discussion of proximity graphs and their applications, we refer the reader to the survey papers by Toussaint [20], [22]. For an application of the relative neighborhood graph to wireless networks, see [11].

To the best of the authors' knowledge, random Yao graphs have not been studied previously. Although researchers have been interested in spanners having small maximum degree (see the textbook by Narasimhan and Smid [15, Chapters 4 and 8] for a survey), most research in this area has been on constructing spanners that have low degree in the worst case. Some of these constructions have been adapted for use in the unit disk graph model of wireless networks [23], but the computation of these spanners is not quite as straightforward and local as that of Yao graphs.

The remainder of this paper is organized as follows. In Section 2 we present our results on Gabriel graphs. In Section 3 we present our results on Yao graphs. Each of these sections concludes with a summary and discussion of possible generalizations and limitations.

\section{Gabriel graphs}

In this section we prove bounds on the maximum degree of vertices in a Gabriel graph. Before we begin, we discuss an equation that is central to all our upper and lower bounds, as well as many other bounds of this type.

Let $c>0$ be a constant, and let $k=c \log n / \log \log n$. In all our bounds, the value $k^{k}$ appears at some point in the computations. Note that

$$
k^{k}=n^{c(1+(\log c-\log \log \log n) / \log \log n)}=n^{c-o(1)} .
$$

In particular, $k=O\left(n^{c}\right)$ and, for any $\varepsilon>0, k=\Omega\left(n^{c-\varepsilon}\right)$.

\subsection{A lower bound}

In this section we prove the following result.

Theorem 2.1. For a random Gabriel graph defined on $n$ points drawn independently from the uniform distribution on $[0,1]^{2}$,

$$
\lim _{n \rightarrow \infty} \operatorname{Pr}\left\{\text { maximum degree }<\frac{c \log n}{\log \log n}\right\}=0
$$

for all $c<\frac{1}{12}$.

Proof. We start with a technical construction of a region and then a point configuration. This construction is parameterized by an integer $k$ and a positive number $r$. Define the angle $\xi=2 \pi /(3 k)$, and partition the plane into $3 k$ sectors of angle $\xi$ each, with center at the origin. We refer the reader to Figure 3 for further explanations.

Draw two concentric circles, both centered at the origin, of radii $r$ and $R$ with $R>r$, so that $r=R \cos \xi$. If the sectors are numbered $C_{1}, C_{2}, \ldots, C_{3 k}$ (clockwise) and the circles are $S_{r}$ and $S_{R}$, then we mark $k$ regions (shown in dark gray in Figure 3). These regions are of the form $\left(S_{R} \backslash S_{r}\right) \cap\left(C_{3 i+1} \cup C_{3 i+2}\right)$ for $0 \leq i \leq k-1$. Call these regions pearl regions and denote 


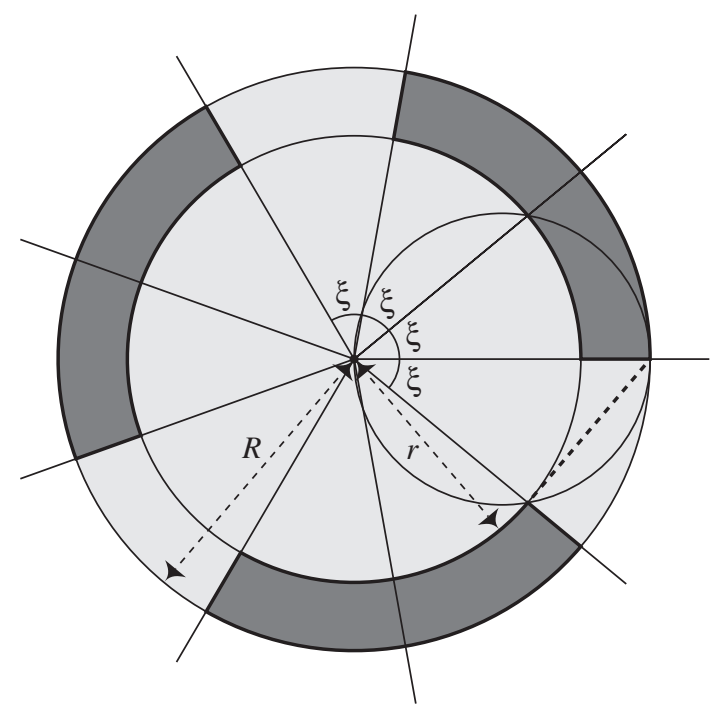

FIGURE 3: The definition of a pearl.

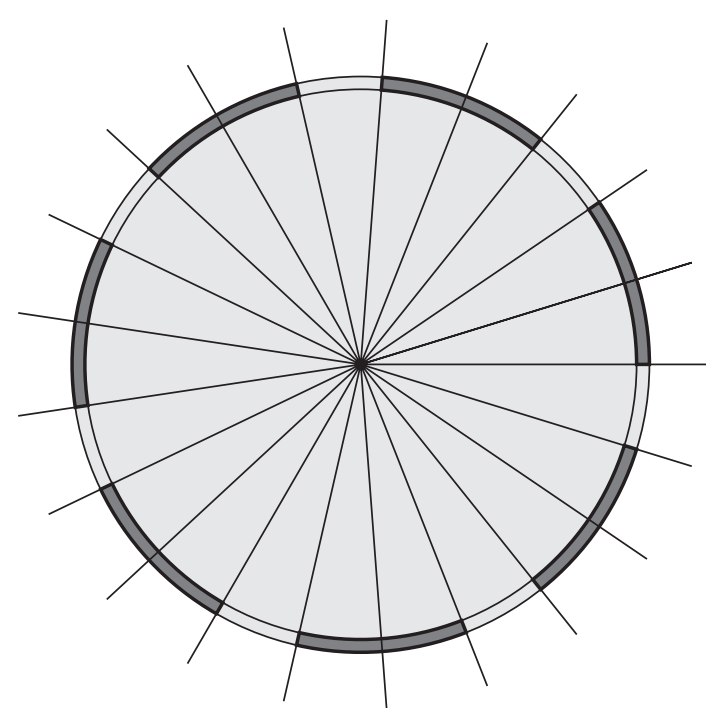

FIGURE 4: The shaded regions define a $(7, r)$-tiara.

them by $P_{1}, \ldots, P_{k}$. Any circle with its diameter being the segment linking the origin with any point in a pearl region totally avoids any other pearl region. To see this, refer to Figure 3 and recall that $r=R \cos \xi$.

Assume that we are given $m$ points in the plane, $x_{1}, \ldots, x_{m}$, and a center $x$. If $x+A$ denotes the translate of a set $A$ by $x$ then we call $x$ a $(k, r)$-tiara for $x_{1}, \ldots, x_{m}$ if exactly $k$ of the points $x_{i}$ fall in $x+S_{R}$, and if each set $x+P_{j}$ covers exactly one of these $x_{i}$ s. See Figure 4 . If we construct the Gabriel graph for $x, x_{1}, \ldots, x_{m}$ then the degree of the vertex at $x$ is at least $k$ if $x$ is a $(k, r)$-tiara for $x_{1}, \ldots, x_{m}$. 
The above construction and definitions are for any point sets. Assume that a random sample of size $n$ is drawn from the uniform distribution on $[0,1]^{2}$, and denote it by $X_{1}, \ldots, X_{n}$. Define $k=\max (3,\lfloor c \log n / \log \log n\rfloor)$ and $r=1 / \sqrt{n}$. We say that $X_{i}$ is a jewel if $X_{i}$ is a $(k, r)$-tiara for $\left\{X_{j}: j \neq i\right\}$ and if $X_{i}$ is a distance at least $2 r$ from the perimeter of $[0,1]^{2}$. Note that $R \leq 2 r$, so that $X_{i}+P_{j} \subseteq[0,1]^{2}$ for all $j$.

We compute the probability that $X_{1}$ is a jewel given $X_{1}=x$, provided that $x$ is a distance at least $2 r$ from the perimeter of the unit square. Note that this probability may be written as a multinomial probability. If $p$ is the area of $x+P_{j}$, we have, in particular,

$$
\begin{aligned}
\operatorname{Pr}\left\{X_{1} \text { is a jewel } \mid X_{1}=x\right\} & =\frac{(n-1) !}{(n-1-k) !} p^{k}\left(1-\pi R^{2}\right)^{n-1-k} \\
& =\frac{(n-1) !}{(n-1-k) !} p^{k}\left(1-\frac{\pi}{n \cos ^{2} \xi}\right)^{n-1-k} \\
& \geq \frac{(n-1) !}{(n-1-k) !} p^{k}\left(1-2 \frac{\pi}{n}\right)^{n-1-k} \quad(\text { since } \xi \leq 2 \pi / 9<\pi / 4) \\
& \geq(n-k)^{k} p^{k}\left(1-\frac{2 \pi}{n}\right)^{n} \\
& \geq(n-k)^{k} p^{k}\left(\frac{1}{3}\right)^{2 \pi} .
\end{aligned}
$$

As $k \geq 3$, we have $\xi \leq 2 \pi / 9<1$, so that $\tan \xi \geq \xi$. Therefore,

$$
p=\left(R^{2}-r^{2}\right) \xi=R^{2} \xi \sin ^{2} \xi=r^{2} \xi \tan ^{2} \xi \geq \frac{\xi^{3}}{n} .
$$

Resubstitution yields

$$
\begin{aligned}
\operatorname{Pr}\left\{X_{1} \text { is a jewel } \mid X_{1}=x\right\} & \geq(n-k)^{k} \frac{\xi^{3 k}}{n^{k}}\left(\frac{1}{3}\right)^{2 \pi} \\
& =\left(1-\frac{k}{n}\right)^{k}\left(\frac{1}{3}\right)^{2 \pi} \xi^{3 k} \\
& =\left(1-\frac{k}{n}\right)^{k}\left(\frac{1}{3}\right)^{2 \pi}\left(\frac{2 \pi}{3}\right)^{3 k} k^{-3 k} \\
& \geq k^{-3 k}
\end{aligned}
$$

when $n$ is large enough, uniformly over all $x$ a distance at least $2 r$ from the perimeter of the unit square. We may now uncondition. If $N$ is the number of jewels among the data points, we have

$$
\mathrm{E}[N]=n \operatorname{Pr}\left\{X_{1} \text { is a jewel }\right\} \geq n(1-4 r)^{2} k^{-3 k} \sim n k^{-3 k} .
$$

If $k$ is as we picked it, and $c<\frac{1}{3}$, then $\mathrm{E}[N] \rightarrow \infty$. This is not quite enough to show that $\operatorname{Pr}\{N>$ $0\} \rightarrow 1$. There are several routes we can follow at this point: we could Poissonize the sample size; we might redefine jewels so that at most one jewel occurs in any region of a regular grid. Both tricks create enough independence to get by. Instead, we opt to use the second moment method (for references, see [1, Chapter 4]). When applied to a counting random variable $N=\sum_{i=1}^{n} Y_{i}$, where the $Y_{i}$ s are $\{0,1\}$-valued with a permutation-invariant joint distribution, the second moment method implies that $N / \mathrm{E}[N] \rightarrow 1$ in probability whenever $\mathrm{E}[N] \rightarrow \infty$ and

$$
\limsup _{n \rightarrow \infty} \frac{\mathrm{E}\left[Y_{1} Y_{2}\right]}{\mathrm{E}\left[Y_{1}\right] \mathrm{E}\left[Y_{2}\right]} \leq 1 .
$$


In our case we only need to verify the latter condition when $Y_{i}$ is the indicator that $X_{i}$ is a jewel, so that $N$ is the number of jewels. Let $A$ be the event that $X_{1}$ or $X_{2}$ is within $2 r$ of the perimeter of the unit square, or that $\left\|X_{1}-X_{2}\right\| \leq 4 r$. On $A^{\mathrm{c}}$, the complement of $A$, we have, by the multinomial argument given above, but now applied to two tiaras,

$$
\mathrm{E}\left[Y_{1} Y_{2} \mid A^{\mathrm{c}}\right]=\frac{(n-2) !}{(n-2-2 k) !} p^{2 k}\left(1-2 \pi R^{2}\right)^{n-2-2 k},
$$

where $p$ is the area of a pearl region $P_{j}$. We recall that

$$
\mathrm{E}\left[Y_{1}\right] \geq \frac{(n-1) !}{(n-1-k) !} p^{k}\left(1-\pi R^{2}\right)^{n-1-k} \geq k^{-3 k}
$$

for large enough $n$. Thus, for such large $n$,

$$
\begin{aligned}
\frac{\mathrm{E}\left[Y_{1} Y_{2}\right]}{\mathrm{E}\left[Y_{1}\right] \mathrm{E}\left[Y_{2}\right]} & =\frac{\operatorname{Pr}\{A\} \mathrm{E}\left[Y_{1} Y_{2} \mid A\right]}{\mathrm{E}\left[Y_{1}\right] \mathrm{E}\left[Y_{2}\right]}+\frac{\operatorname{Pr}\left\{A^{\mathrm{c}}\right\} \mathrm{E}\left[Y_{1} Y_{2} \mid A^{\mathrm{c}}\right]}{\mathrm{E}\left[Y_{1}\right] \mathrm{E}\left[Y_{2}\right]} \\
& \leq \frac{\operatorname{Pr}\{A\}}{\mathrm{E}\left[Y_{1}\right] \mathrm{E}\left[Y_{2}\right]}+\frac{\mathrm{E}\left[Y_{1} Y_{2} \mid A^{\mathrm{c}}\right]}{\mathrm{E}\left[Y_{1}\right] \mathrm{E}\left[Y_{2}\right]} \\
& \leq \frac{8 r+16 \pi r^{2}}{\mathrm{E}\left[Y_{1}\right] \mathrm{E}\left[Y_{2}\right]}+\frac{(n-2) !}{(n-2-2 k) !} p^{2 k}\left(1-2 \pi R^{2}\right)^{n-2-2 k} \\
& \times \frac{(n-1-k) !^{2}}{(n-1) !^{2}} p^{-2 k}\left(1-\pi R^{2}\right)^{2 k+2-2 n} \\
& \leq \frac{67}{n^{1 / 2} k^{-6 k}}+1+O\left(\frac{k^{2}}{n}\right) .
\end{aligned}
$$

We are done if $n^{1 / 2} k^{-6 k} \rightarrow \infty$. For this, in the definition of $k$, we need only pick $6 c<\frac{1}{2}$, or $c<\frac{1}{12}$. We have thus shown that $N / \mathrm{E}[N] \rightarrow 1$ in probability when $c<\frac{1}{12}$ in the definition of $k$. We conclude that $\operatorname{Pr}\{N=0\} \rightarrow 0$ for such choices of $c$. Therefore,

$$
\lim _{n \rightarrow \infty} \operatorname{Pr}\left\{\text { maximum degree in Gabriel graph }<\frac{c \log n}{\log \log n}\right\}=0
$$

for all $c<\frac{1}{12}$.

\subsection{An upper bound}

Theorem 2.2. For a random Gabriel graph defined on $n$ points drawn independently from the uniform distribution on $[0,1]^{2}$,

$$
\lim _{n \rightarrow \infty} \operatorname{Pr}\left\{\text { maximum degree }>\frac{c \log n}{\log \log n}\right\}=0
$$

for all $c>1$.

Proof. At a point $x$, partition the space into $k$ equal sectors each having their apex at $x$ and of angle $2 \pi / k$ each, where $k=\lceil\sqrt{\log n}\rceil$. Within each sector, we color the point nearest to $x$ red. If a sector has a red point $y$, consider the perpendicular line at $y$ to the segment $(y, x)$ and call this line the separator. All points in the same sector, on the same side of the separator as $x$ are colored blue. In Figure 5, these are precisely the points that fall in the shaded wedges. 


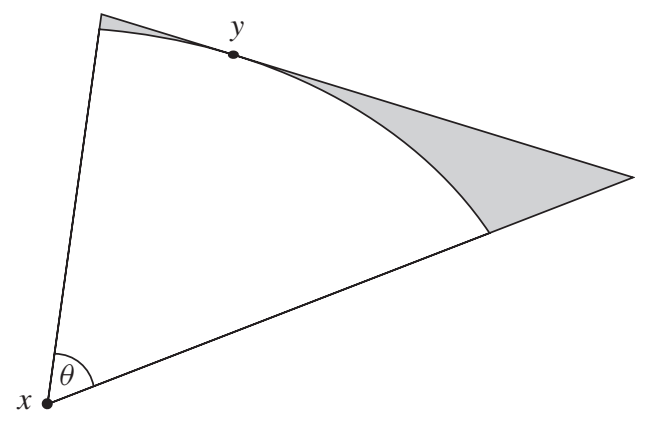

Figure 5: The definition of a wedge.

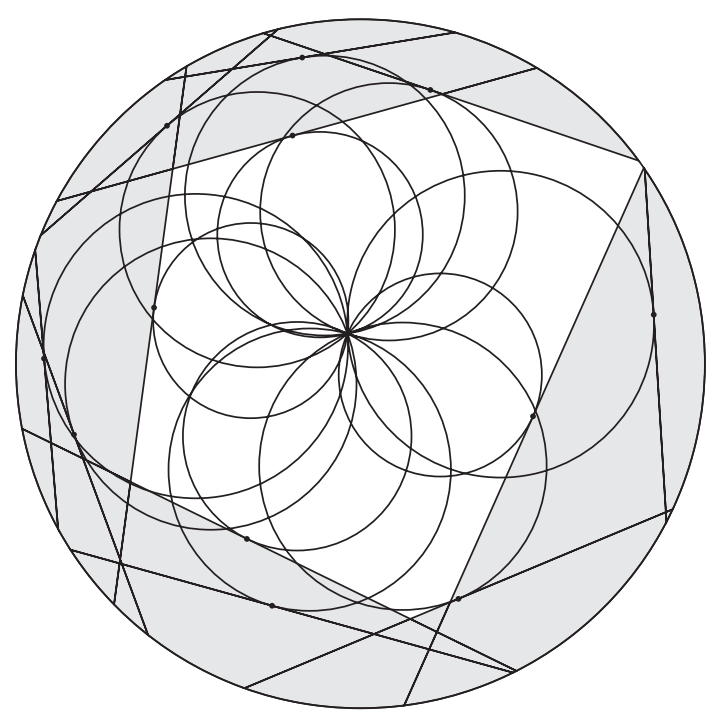

FIGURE 6: Several points and their separators.

We first claim that each Gabriel graph neighbor of $x$ is colored red or blue. Indeed, any point $y$ excludes all points at the other side of the separator-the side that does not contain $x$. Thus, if there is a red point in the sector, only blue points can possibly be Gabriel neighbors of $x$. Let $r=3 \sqrt{\log n / n}$ and recolor any (blue or red) point $z$ to be yellow if it is a Gabriel neighbor of $x$ and $\|x z\|>r$.

Figure 6 shows several points with their separators. No point in the shaded area can be a Gabriel neighbor of the point $x$ at the origin. Note that, for every point in the shaded area, the Gabriel circle through the origin contains another point. Figure 7 shows several sectors and red points, together with the wedges in which blue points must fall. The angle of each sector is $\theta=2 \pi / k$.

Let $N_{\mathrm{r}}, N_{\mathrm{b}}$, and $N_{\mathrm{y}}$ be the total number of red, blue, and yellow points, respectively. Recall that $k=\lceil\sqrt{\log n}\rceil=o(\log n / \log \log n)$, so $N_{\mathrm{r}} \leq k=o(\log n / \log \log n)$. Also, conditioning 


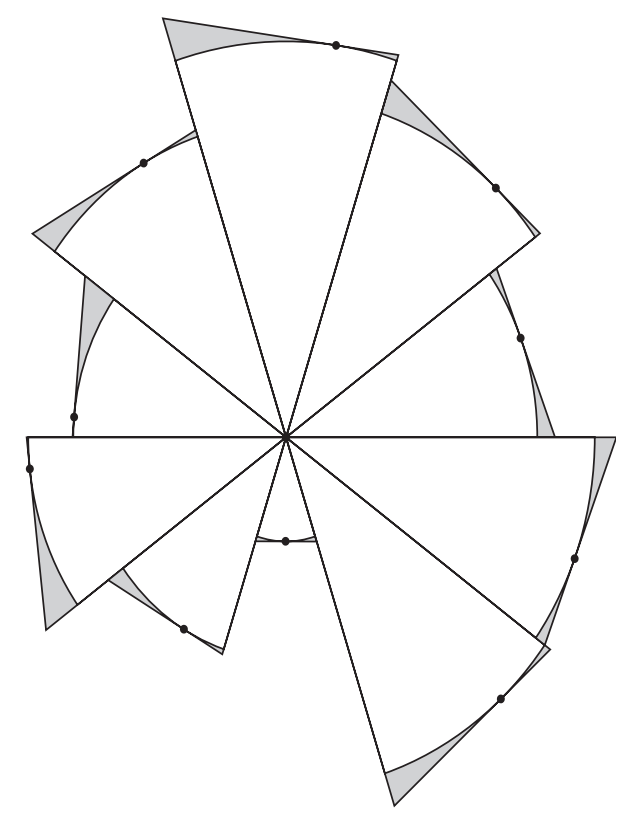

FIGURE 7: Sectors, red points, and blue wedges.

on $X_{1}=x$,

$$
\begin{aligned}
\mathrm{E}\left[N_{\mathrm{y}} \mid X_{1}=x\right] & =n \operatorname{Pr}\left\{X_{2} \text { is a Gabriel neighbor of } X_{1},\left\|X_{2}-X_{1}\right\| \geq r \mid X_{1}=x\right\} \\
& \leq n\left(1-\pi(r / 2)^{2} / \pi\right)^{n-1}
\end{aligned}
$$

(because at least a $\frac{1}{4}$ fraction of the Gabriel circle through $x$ and $X_{2}$ falls in the unit square)

$$
\begin{aligned}
& \leq n\left(1-r^{2} / 4\right)^{n-1} \\
& \leq n \mathrm{e}^{-9(n-1) \log n /(4 n)} \\
& \sim n^{1-9 / 4} \\
& \rightarrow 0 .
\end{aligned}
$$

Thus, it suffices to study $N_{\mathrm{b}}$.

As $\tan \theta \leq \theta+\theta^{3}$ for $0 \leq \theta \leq 1$, the area of each wedge is at most

$$
\begin{aligned}
\frac{r^{2}}{2}(\tan \theta-\theta) & \leq \frac{9 \log n \theta^{3}}{2 n} \\
& \leq \frac{72 \log n \pi^{3}}{2 n k^{3}} \\
& \leq \frac{1200 \log n}{n k^{3}}
\end{aligned}
$$

The total wedge area around $X_{1}=x$ is thus not more than

$$
\frac{1200 \log n}{n k^{2}} \leq \frac{1200}{n} \text {. }
$$


Given $X_{1}=x$ and the collection of red points, the $n-1-N_{\mathrm{r}}$ other points are uniformly distributed on the unit square, but not in any of the $N_{\mathrm{r}}$ circular sectors just inside the wedges, and not in any circular sectors of radius $r$ defined when no red point is present in the sector. Call the density $f$ and its support set $S$. Clearly, $1 \leq f \leq 1 /\left(1-\pi r^{2}\right)$. Of the $n-1-N_{\text {r points, let }}$ $M$ denote the total number of points falling in the wedges. Clearly, $M$ is stochastically smaller than a binomial random variable with parameters $m=n-1-N_{\mathrm{r}}$ and $p=1200 / n\left(1-\pi r^{2}\right)$. In particular, using $l ! \geq(l / \mathrm{e})^{l}$, and letting Pr denote the conditional probability,

$$
\begin{aligned}
\operatorname{Pr}\{M \geq l\} & \leq \sum_{j=l}^{m}\left(\begin{array}{c}
m \\
j
\end{array}\right) p^{j}(1-p)^{m-j} \\
& \leq \sum_{j=l}^{\infty} \frac{(m p)^{j}}{j !} \\
& =\frac{(m p)^{l}}{l !} \sum_{j=0}^{\infty} \frac{(m p)^{j} l !}{(l+j) !} \\
& \leq \frac{(m p)^{l}}{l !} \sum_{j=0}^{\infty}\left(\frac{m p}{l}\right)^{j} \\
& =\frac{(m p)^{l}}{l !(1-m p / l)} \\
& \leq \frac{(m p \mathrm{e} / l)^{l}}{(1-m p / l)} \\
& \leq \frac{(n p \mathrm{e} / l)^{l}}{(1-n p / l)} .
\end{aligned}
$$

We set $l=\lceil c \log n / \log \log n\rceil$ for a constant $c$ and note that $n p / l=o(1)$. By the union bound, the probability that there exists a point $x$ for which the number of blue points in the wedge collection for $x$ is greater than or equal to $l$ does not exceed

$$
n \times \frac{(n p \mathrm{e} / l)^{l}}{(1-n p / l)} .
$$

As $n p=1200+o(1)$, the above expression is, for all large enough $n$, not more than $2 n(3600 / l)^{l}$. By (2.1), this tends to 0 when $c>1$.

The probability that, for one of the data points, $N_{\mathrm{y}}>0$ is not more than

$$
n \times(1+o(1)) n^{1-9 / 4} \rightarrow 0 .
$$

Thus, we have shown that, for $c>1$, the probability that the maximum degree exceeds $k+l$ tends to 0 . As $k+l \sim l$, we are done.

\subsection{Remarks}

Higher dimensions. Just as Bern et al. [3] showed for the expected maximum degree in a Delaunay triangulation, the results of Theorems 2.1 and 2.2 extend easily to $\mathbb{R}^{d}$. In particular, for any $d$, there exist constants $a>0$ and $b<\infty$ depending only upon $d$ such that

$$
\lim _{n \rightarrow \infty} \operatorname{Pr}\left\{\text { maximum degree } \notin\left(\frac{a \log n}{\log \log n}, \frac{b \log n}{\log \log n}\right)\right\}=0 .
$$


Edge lengths. The results on $N_{\mathrm{y}}$ in the proof above show that the expected number of Gabriel edges of length at least $3 \sqrt{\log n / n}$ is $o(1)$. Hence, the probability that the maximum edge length exceeds $3 \sqrt{\log n / n}$ tends to 0 . Bounds on sums of functions of the edge lengths of random Gabriel graphs are given in [17].

\section{Yao graphs}

In this section we present our results on Yao graphs. For simplicity, we consider $\theta$-Yao graphs with $\theta=\pi / 2$. The modifications required for other (smaller) values of $\theta$ are discussed at the end of this section. The lower bound in Section 3.1 is obtained using a construction and argument similar to the pearl used to prove Theorem 2.1. The upper bound in Section 3.2 uses different arguments based on maxima.

For the upper bound, we change the distribution model slightly by rotating it by $\pi / 4$. More precisely, let $\mathbb{D}^{2}$ denote the unit square rotated by $\pi / 4$. The upper bound assumes that points are distributed uniformly and independently in $\mathbb{D}^{2}$. At the end of this section, we discuss why this slightly different assumption is necessary.

\subsection{A lower bound}

Our lower bound argument is similar to that used for Gabriel graphs, in that we define a configuration of points whose existence implies a vertex of degree $k$ and show that, with high probability, this configuration exists in a random point set.

Theorem 3.1. For a random $\pi / 2$-Yao graph defined by $n$ points drawn independently from the uniform distribution on $[0,1]^{2}$,

$$
\lim _{n \rightarrow \infty} \operatorname{Pr}\left\{\text { maximum degree }<\frac{c \log n}{\log \log n}\right\}=0
$$

for all $c<\frac{1}{8}$.

Proof. Refer to Figure 8(a). Let $r>0$ be a real number, and let $k$ be a positive integer. Define $k$ square regions $P_{1}, \ldots, P_{k}$, where

$$
P_{i}=\left[\frac{(i-1) r}{k}, \frac{i r}{k}\right] \times\left[r-\frac{i r}{k}, r-\frac{(i-1) r}{k}\right]
$$

These regions are called steps.

Assume that we are given $m$ points in the plane, $x_{1}, \ldots, x_{m}$, and a center $x$. Then we call $x$ a $(k, r)$-staircase for $x_{1}, \ldots, x_{m}$ if exactly $k$ of the points $x_{i}$ fall into the square $x+[-r, r]^{2}$ and if each step $x+P_{j}$ covers exactly one of these $x_{i}$ s. If we construct the $\pi / 2$-Yao graph for $x, x_{1}, \ldots, x_{m}$ and $x$ is a $(k, r)$-staircase for $x_{1}, \ldots, x_{m}$, then every point in each of the $k$ steps is adjacent to $x$, so $x$ is a vertex of degree at least $k$ (see Figure 8(b)).

Let $k=c \log n / \log \log n$, let $r=\sqrt{2 / n}$, and let $X_{1}, \ldots, X_{n}$ be $n$ points drawn uniformly and independently from $[0,1]^{2}$. Then the area, $p$, of a step in a $(k, r)$-staircase is 


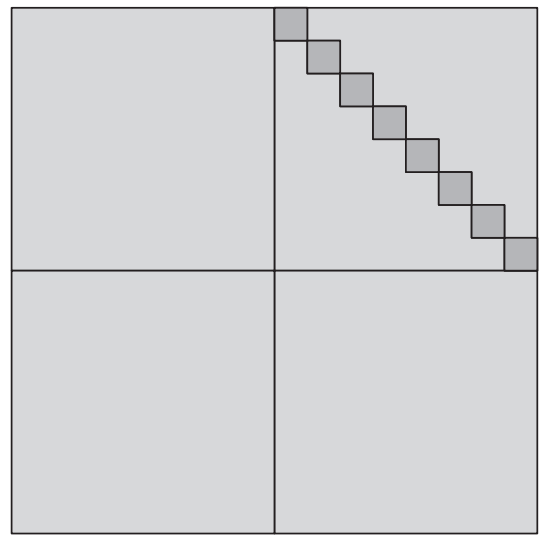

(a)

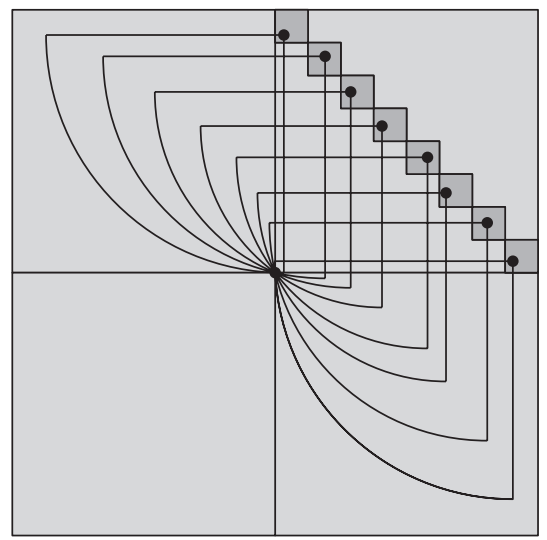

(b)

Figure 8: An $(8, r)$-staircase.

$p=(r / k)^{2}=2 / n k^{2}$, so

$$
\begin{aligned}
\operatorname{Pr}\left\{X_{1} \text { is a }(k, r) \text {-staircase } \mid X_{1} \in[r, 1-r]^{2}\right\} & =\frac{(n-1) !}{(n-k-1) !} p^{k}\left(1-\frac{8}{n}\right)^{n-k-1} \\
& \geq\left(1-\frac{k}{n}\right)^{k}\left(1-\frac{8}{n}\right)^{n} 2^{k} k^{-2 k} \\
& \geq k^{-2 k}
\end{aligned}
$$

for sufficiently large $n$. Thus, if $N$ is the number of staircases among $X_{1}, \ldots, X_{n}$ then

$$
\mathrm{E}[N] \geq n(1-2 r)^{2} k^{-2 k}=\Omega\left(n^{1-2 c-\varepsilon}\right) \rightarrow \infty,
$$

provided that $c<\frac{1}{2}$.

As before, we finish the proof using the second moment method. Let $A$ denote the event that $\left\{X_{1}, X_{2}\right\} \not \subset[r, 1-r]^{2}$ or that $X_{2} \in X_{1}+[-r, r]^{2}$, and let $A^{\mathrm{c}}$ denote the complement of $A$. Let $Y_{i}, i \in\{1,2\}$, denote the indicator variable that $X_{i}$ is a staircase. Then, for sufficiently large $n$,

$$
\begin{aligned}
\frac{\mathrm{E}\left[Y_{1} Y_{2}\right]}{\mathrm{E}\left[Y_{1}\right] \mathrm{E}\left[Y_{2}\right]} & \leq \frac{\mathrm{E}\left[Y_{1} Y_{2}\right]}{k^{-4 k}} \\
& =k^{4 k}\left(\operatorname{Pr}\{A\} \mathrm{E}\left[Y_{1} Y_{2} \mid A\right]+\operatorname{Pr}\left\{A^{\mathrm{c}}\right\} \mathrm{E}\left[Y_{1} Y_{2} \mid A^{\mathrm{c}}\right]\right) \\
& \leq k^{4 k}\left(\left(4 r+4 r^{2}\right)+\operatorname{Pr}\left\{A^{\mathrm{c}}\right\} \mathrm{E}\left[Y_{1} Y_{2} \mid A^{\mathrm{c}}\right]\right) \\
& \leq k^{4 k}\left(\left(4 r+4 r^{2}\right)+\frac{(n-2) !}{(n-2-2 k) !}\left(\frac{1}{n k^{2}}\right)^{2 k}\left(1-\frac{16}{n}\right)^{n-2-2 k}\right) \\
& \leq k^{4 k}\left(\left(4 r+4 r^{2}\right)+n^{2 k}\left(\frac{1}{n k^{2}}\right)^{2 k}\left(1-\frac{16}{n}\right)^{n-2-2 k}\right) \\
& \leq k^{4 k}\left(4 \sqrt{\frac{2}{n}}+\frac{8}{n}\right)+1 \\
& =1+O\left(n^{4 c-1 / 2}\right),
\end{aligned}
$$

so $\lim _{n \rightarrow \infty} \mathrm{E}\left[Y_{1} Y_{2}\right] / \mathrm{E}\left[Y_{1}\right] \mathrm{E}\left[Y_{2}\right]=1$ for any $c<\frac{1}{8}$. 


\subsection{An upper bound}

Next we prove an upper bound on the maximum degree in a $(\pi / 2)$-Yao graph. The upper bound is based on the observation that the neighbors of a node in a Yao graph are so-called minima. Let $x_{1}, \ldots, x_{n}$ be a set of points. We say that a point $x_{i}$ dominates $x_{j}$ if the $x$ and $y$ coordinates of $x_{i}$ are larger than the $x$ and $y$ coordinates of $x_{j}$, respectively. A point $x$ is maximal with respect to $x_{1}, \ldots, x_{n}$ if $x$ is not dominated by any $x_{i}$. A point $x$ is minimal if $x$ does not dominate any point $x_{i}$.

Before we can present the upper bound, we require a few preliminary results about maxima and minima. First, though, we recall a classic result obtained using Chernoff's bounding method [5].

Lemma 3.1. Let $Y_{1}, \ldots, Y_{m}$ be a sequence of independent $\{0,1\}$-valued random variables, let $Y=\sum_{i=1}^{m} Y_{i}$, and let $\mu=\mathrm{E}[Y]$. Then, for any $\delta>0$,

$$
\operatorname{Pr}\{Y>(1+\delta) \mu\} \leq\left(\frac{\mathrm{e}^{\delta}}{(1+\delta)^{(1+\delta)}}\right)^{\mu} .
$$

The following result is already quite well known. We include a proof sketch only for the sake of completeness.

Lemma 3.2. Let $X_{1}, \ldots, X_{m}$ be a sequence of points drawn independently and uniformly from a rectangle $[a, b] \times[c, d]$ having area greater than 0 , and let $M$ be the number of maximal (or minimal) points among $X_{1}, \ldots, X_{m}$. Then, for any $\delta>0$,

$$
\log m \leq \mathrm{E}[M] \leq \log m+1
$$

and

$$
\operatorname{Pr}\{M>(1+\delta) \mathrm{E}[M]\} \leq\left(\frac{\mathrm{e}^{\delta}}{(1+\delta)^{1+\delta}}\right)^{\log m}
$$

Proof. Sort the elements of $X_{1}, \ldots, X_{m}$ by decreasing $x$ coordinate, so that $X_{i}$ is maximal if and only if its $y$ coordinate is the maximum among the $y$ coordinates of $X_{1}, \ldots, X_{i}$. Let $Y_{i}=1$ if $X_{i}$ is maximal and $Y_{i}=0$ otherwise. Obviously, $\mathrm{E}\left[Y_{i}\right]=1 / i$, so

$$
\mathrm{E}[M]=\mathrm{E}\left[\sum_{i=1}^{m} Y_{i}\right]=\sum_{1=1}^{m} \frac{1}{i} .
$$

The inequality $\log m \leq \mathrm{E}[M] \leq \log m+1$ is then obtained by bounding the above harmonic sum using the integral $\int_{1}^{m}(1 / x) \mathrm{d} x$ (see, e.g. [6, Appendix A.2]).

To prove the second part of the lemma, we use the fact that the random variables $Y_{1}, \ldots, Y_{m}$ are independent [7], [9]. The result then follows immediately from Lemma 3.1.

Unfortunately, the points we consider will not always be drawn from a rectangle. A $t$-shape is a closed maximal subset of $\mathbb{R}^{2}$ that is bounded by the $x$ and $y$ axes and a $y$-monotone polygonal path consisting of at most $t$ edges (a piecewise-linear function of $y$ having at most $t$ pieces). See Figure 9(a).

Lemma 3.3. Let $X_{1}, \ldots, X_{m}$ be a sequence of points drawn independently and uniformly from a $t$-shape $S$ having area greater than 0 , and let $M$ be the number of minimal points among $X_{1}, \ldots, X_{m}$. Then, for any $\delta>0$,

$$
\mathrm{E}[M] \leq 2 t(\log m+1)
$$




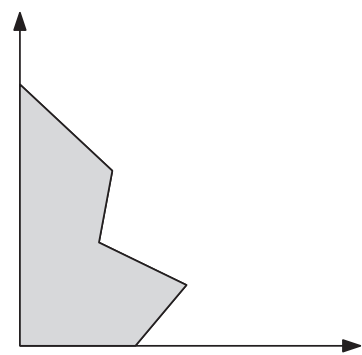

(a)

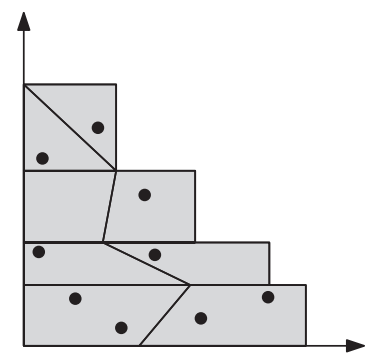

(b)

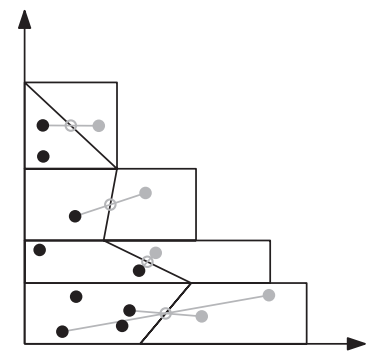

(c)

Figure 9: (a) A $t$-shape $S$. (b) Covering $S$ to obtain a shape $S^{\prime}$ and uniformly distributing points in $S^{\prime}$. (c) Reflecting the points in $S^{\prime}$ to obtain points uniformly distributed in $S$.

and

$$
\operatorname{Pr}\{M>(1+\delta) 2 t(\log m+1)\} \leq 2 t\left(\frac{\mathrm{e}^{\delta}}{(1+\delta)^{1+\delta}}\right)^{\log m} .
$$

Proof. Cover $S$ with at most $t$ rectangles $R_{1}, \ldots, R_{\ell}, \ell \leq t$, whose total area is twice the area of $S$, as shown in Figure 9(b). Let $S^{\prime}=\bigcup_{i=1}^{\ell} R_{i}$ be the resulting subset of $\mathbb{R}^{2}$. Generate points $Z=\left\{Z_{1}, \ldots, Z_{m}\right\}$ uniformly and independently in $S^{\prime}$. For each point $Z_{i}$ in $R_{j}$, if $Z_{i} \in S$ then set $X_{i}=Z_{i}$. Otherwise, set $X_{i}$ to be the reflection of $Z_{i}$ through the center of $R_{j}$. Observe that $X_{1}, \ldots, X_{m}$ are uniformly distributed in $S$. Furthermore, if $X_{i} \in R_{j}$ is minimal with respect to $X_{1}, \ldots, X_{m}$ then $Z_{i}$ is either maximal or minimal with respect to $Z \cap R_{j}$. Therefore, if $M_{j}$ denotes the number of minimal elements of $X$ contained in $R_{j}$ then, by the first part of Lemma 3.2, $\mathrm{E}\left[M_{j}\right] \leq 2(\log m+1)$ and

$$
\mathrm{E}[M]=\mathrm{E}\left[\sum_{j=1}^{t} M_{j}\right] \leq 2 t(\log m+1) .
$$

By applying the second part of Lemma $3.2 t$ times, and using the union bound, we also obtain

$$
\operatorname{Pr}\{M>(1+\delta) 2 t(\log m+1)\} \leq 2 t\left(\frac{\mathrm{e}^{\delta}}{(1+\delta)^{1+\delta}}\right)^{\log m},
$$

as required.

We now have all the tools required to prove our upper bound.

Theorem 3.2. For a random ( $\pi / 2)$-Yao graph defined on $n$ points drawn independently from the uniform distribution on $\mathbb{D}^{2}$,

$$
\lim _{n \rightarrow \infty} \operatorname{Pr}\left\{\text { maximum degree }>\frac{4 c \log n}{\log \log n}\right\}=0
$$

for all $c>4$.

Proof. Let $X_{1}, \ldots, X_{n}$ be points uniformly and independently distributed in $\mathbb{D}^{2}$, and let $G$ be the $(\pi / 2)$-Yao graph of $X_{1}, \ldots, X_{n}$. Let $\ell=\sqrt{d \log n / n}$. We will first consider the edges of $G$ whose length is at most $\ell$. Consider the square $S=X_{1}+[0, \ell]^{2}$, which contains all 
neighbors of $X_{1}$ in $X_{1}$ 's 1-cone. Let $N$ denote the number of points of $X_{2}, \ldots, X_{n}$ contained in $S$. Then $\mathrm{E}[N] \leq n \ell^{2}=d \log n$ and, by Lemma 3.1,

$$
\operatorname{Pr}\{N>2 d \log n\} \leq\left(\frac{\mathrm{e}}{4}\right)^{d \log n}=n^{d(1-\log 4)} .
$$

Let $N^{\prime}$ denote the number of points in $S$ that are neighbors of $X_{1}$ in the Yao graph. Each such point is minimal with respect to the $N$ points of $X_{2}, \ldots, X_{n}$ contained in $S$. Furthermore, $S \cap \mathbb{D}^{2}$ is a $t$-shape for $t \leq 2$. By the first part of Lemma 3.3, conditioned on $N \leq 2 d \log n$, the expected number of minimal points, and, hence, the number of neighbors of $X_{1}$ in $S$, is small:

$$
\mathrm{E}\left[N^{\prime} \mid N \leq 2 d \log n\right] \leq 4(\log (2 d \log n)+1)=4 \log \log n+\Theta(1) .
$$

Define $v=\log (2 d \log n)$ and let $k=(c \log n) /(\log \log n)$. By the second part of Lemma 3.3, with $t=2$,

$$
\begin{aligned}
\operatorname{Pr}\left\{N^{\prime}>k \mid N \leq 2 d \log n\right\} & =\operatorname{Pr}\left\{N^{\prime}>\frac{c \log n}{4(v+1)(\log \log n)}(4(v+1)) \mid N \leq 2 d \log n\right\} \\
& \leq 4\left(\frac{f(n)}{g(n)}\right)^{\log (2 d \log n)} \\
& \leq 4\left(\frac{f(n)}{g(n)}\right)^{\log \log n},
\end{aligned}
$$

where

$$
\begin{aligned}
f(n)^{\log \log n} & =\exp \left(\frac{c \log n}{4(v+1)(\log \log n)}-1\right)^{\log \log n} \\
& \leq \exp \left(\frac{c \log n}{4(v+1)}-1\right) \\
& =n^{o(1)}
\end{aligned}
$$

and

$$
\begin{aligned}
g(n)^{\log \log n} & =\left(\left(\frac{c \log n}{4(v+1)(\log \log n)}\right)^{c \log n / 4(v+1)(\log \log n)}\right)^{\log \log n} \\
& =\left(\frac{c \log n}{4(v+1)(\log \log n)}\right)^{c \log n / 4(v+1)} \\
& =\Omega\left(n^{c / 4-\varepsilon}\right)
\end{aligned}
$$

for any $\varepsilon>0$. Putting this all together, we obtain

$$
\operatorname{Pr}\left\{N^{\prime}>k \mid N \leq 2 d \log n\right\} \leq \frac{n^{o(1)}}{\Omega\left(n^{c / 4-\varepsilon}\right)}=O\left(n^{-c / 4+\varepsilon}\right) .
$$

Unconditioning, we obtain

$$
\operatorname{Pr}\left\{N^{\prime}>k\right\}=O\left(n^{-c / 4+\varepsilon}+n^{d(1-\log 4)}\right) .
$$




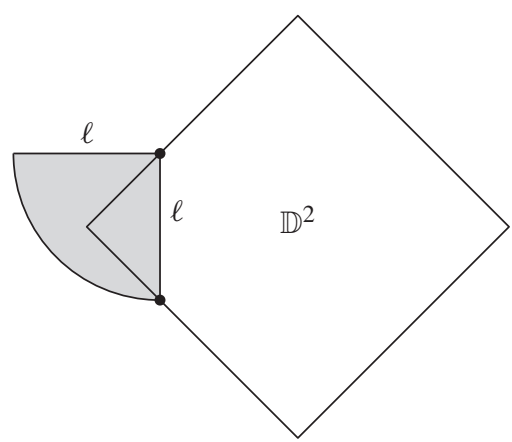

Figure 10: An edge of length $\ell$ defines an empty subset of $\mathbb{D}^{2}$ whose area is at least $(\ell / 2)^{2}$.

Let $G^{\prime}$ be the subgraph of $G$ consisting only of edges of length at most $\ell$, and let $D^{\prime}$ denote the maximum degree of a vertex in $G^{\prime}$. Repeating the above argument $4 n$ times and using the union bound gives

$$
\operatorname{Pr}\left\{D^{\prime}>4 k\right\}=O\left(n^{1-c / 4+\varepsilon}+n^{1+d(1-\log 4)}\right) .
$$

Finally, all that remains is to argue that $G$ has no edges of length greater than $\ell$. An edge of length at least $\ell$ defines an empty region of area at least $\pi \ell^{2} / 4$. For $\ell<\frac{1}{2}$, a portion of this empty region whose area is at least $(\ell / 2)^{2}$ is contained in $\mathbb{D}^{2}$ (see Figure 10). Therefore, the probability of there being any edge of length greater than $\ell=\sqrt{d \log n / n}$ is at most

$$
\begin{aligned}
4 n\left(1-\ell^{2} / 4\right)^{n-2} & =4 n\left(1-\frac{d \log n}{4 n}\right)^{n-2} \\
& \leq 4 n \mathrm{e}^{-(n-1) d \log n / 4 n} \\
& \leq 4 n^{1-(n-1) d / 4 n} \\
& =4 n^{1-(1-1 / n) d / 4} .
\end{aligned}
$$

At last, let $D$ be the maximum degree of any vertex in $G$. Putting everything together, we obtain

$$
\operatorname{Pr}\{D>4 k\}=O\left(n^{1-c / 4+\varepsilon}+n^{1+d(1-\log 4)}+n^{1-(1-1 / n) d / 4}\right) \rightarrow 0
$$

for any $c>4$ and $d>\max \{1 /(\log 4-1), 4\}$.

\subsection{Remarks}

Why $\mathbb{D}^{2}$ ? The proof of Theorem 3.2 actually shows that the probability that $G$ has a vertex of degree more than $c \log n / \log \log n$ is $n^{-\Omega(c)}$. The last step in the proof requires that any edge of length $\ell$ defines a portion of the support set of area $\Omega\left(\ell^{2}\right)$ that is empty of points. This is true when the support set is $\mathbb{D}^{2}$, but not true when the support set is the unit square $[0,1]^{2}$. Indeed, the proof breaks down for points drawn from the unit square since, with probability $1 / n$, some element, say $X_{1}$, simultaneously has the minimum $x$ and $y$ coordinates. In this case, the expected degree of $X_{1}$ is equal to the expected number of minimal elements among $X_{2}, \ldots, X_{n}$, which is, by Lemma 3.2, $\Theta(\log n)$.

In a situation where points are uniformly distributed in the unit square, the upper bound in Theorem 3.2 holds if we consider only the points whose distance from the boundary of the square is at least $\sqrt{d \log n / n}$. 
Smaller values of $\theta$. For any constant value of $\theta \leq \pi / 2$, the upper and lower bounds of Theorem 3.1 and Theorem 3.2 still hold. The arguments are almost identical with the exception that the definitions of a staircase and of minima and maxima are modified to take the value of $\theta$ into account. Although the value of $\theta$ appears in the intermediate calculations, for any constant $\theta$, the constants $c=\frac{1}{8}$ and $c=4$ in Theorem 3.1 and Theorem 3.2 are unchanged (though the constant $4 c$ in Theorem 3.2 becomes $(2 \pi / \theta) c)$. However, as noted above, to prove a version of Theorem 3.2, the support set must be rotated so that the difference in angle between any side of the support set and $i \theta$ for $0 \leq i \leq 2 \pi / \theta$ is lower bounded by a constant.

Higher dimensions. Yao graphs are also defined for point sets in $\mathbb{R}^{d}$. The lower bound of Theorem 3.1 can be extended to show that Yao graphs of $n$ points uniformly and independently distributed in $[0,1]^{d}$ have maximum degree $\Omega(\log n / \log \log n)$. Unfortunately, the proof of the upper bound in Theorem 3.2 does not continue to hold in $\mathbb{R}^{d}$.

\section{References}

[1] Alon, N. And Spencer, J. H. (2000). The Probabilistic Method, 2nd edn. John Wiley, New York.

[2] Barrière, L., Fraigniaud, P., Narayanan, L. and Opatrny, J. (2003). Robust position-based routeing in wireless ad hoc networks with irregular transmission range. Wireless Commun. Mobile Comput. 3, 141-153.

[3] Bern, M., Eppstein, D. And Yao, F. (1991). The expected extremes in a Delaunay triangulation. Internat. J. Comput. Geometry Appl. 1, 79-91.

[4] Bose, P., Morin, P., Stojmenović, I. And Urrutia, J. (2001). Routeing with guaranteed delivery in ad hoc wireless networks. Wireless Networks 7, 609-616.

[5] Chernoff, H. (1952). A measure of the asymptotic efficient of tests of a hypothesis based on the sum of observations. Ann. Math. Statist. 23, 493-507.

[6] Cormen, T. H., Leiserson, C. E., Rivest, R. L. and Stein, C. (2006). Introduction to Algorithms, $2 \mathrm{nd}$ edn. MIT Press, Cambridge, Massachussets.

[7] Devroye, L. (1988). The expected size of some graphs in computational geometry. Comput. Math. Appl. 15, 53-64.

[8] Gabriel, K. R. and SoKal, R. R. (1969). A new statistical approach to geographic variation analysis. Systematic Zoology 18, 259-278.

[9] GLICK, N. (1978). Breaking records and breaking boards. Amer. Math. Monthly 85, 2-26.

[10] Grünewald, M., Lukovszki, T., Schindelhauer, C. And Volbert, K. (2002). Distributed maintenance of resource efficient wireless network topologies. In Proc. 8th Internat. Euro-Par Conf. Parallel Processing, Springer, Berlin, pp. 935-946.

[11] KARP, B. ANd Kung, H. T. (2000). GPSR: greedy perimeter stateless routeing for wireless networks. In Proc. 6th Annual Internat. Conf. Mobile Comput. Networking, ACM, New York, pp. 243-254.

[12] LI, X.-Y., WAN, P.-J. AND WANG, Y. (2001). Power efficient and sparse spanner for wireless ad hoc networks. In IEEE Internat. Conf. Computer Commun. Networks (ICCCN01), Scottsdale, Arizona, pp. 564-567.

[13] Matula, D. W. And Sokal, R. R. (1980). Properties of Gabriel graphs relevant to geographic variation research and the clustering of points in the plane. Geographical Analysis 12, 205-222.

[14] Meester, R. And Roy, R. (1996). Continuum Percolation (Camb. Tracts Math. 119). Cambridge University Press.

[15] Narasimhan, G. and Smid, M. (2007). Geometric Spanner Networks. Cambridge University Press.

[16] Penrose, M. (2003). Random Geometric Graphs (Oxford Stud. Prob. 5). Oxford University Press.

[17] Penrose, M. D. ANd Yukich, Y. E. (2003). Weak laws of large numbers in geometric probability. Ann. Appl. Prob. 13, 277-303.

[18] Schindelhauer, C., Volbert, K. and Ziegler, M. (2007). Geometric spanners with applications in wireless networks. Comput. Geometry 36, 197-214.

[19] TA, X., Mao, G. And Anderson, B. D. O. (2009). On the phase transition width of $K$-connectivity in wireless multihop networks. IEEE Trans. Mobile Comput. 8, 936-949.

[20] Toussaint, G. T. (1980). Pattern recognition and geometrical complexity. In Proc. 5th Internat. Conf. Pattern Recognition, pp. 1324-1347.

[21] Toussaint, G. T. (1980). The relative neighborhood graph of a finite planar set. Pattern Recognition 12, 261-268.

[22] Toussaint, G. T. (1982). Computational geometric problems in pattern recognition. In Pattern Recognition Theory and Applications (NATO Adv. Study Inst. Ser. C: Math. Phys. Sci. 81), Reidel, Dordrecht, eds J. Kittler, K. S. Fu, and L. F. Pau, pp. 73-91. 
[23] WANG, Y. AND LI, X.-Y. (2006). Localized construction of bounded degree and planar spanner for wireless ad hoc networks. ACM Mobile Network Appl. 11, 161-175.

[24] Wattenhofer, R., Li, L., Bahl, P. And WAng, Y. (2001). Distributed topology control for wireless multihop ad hoc networks. In Proc. IEEE INFOCOM, pp. 1388-1397.

[25] YAO, A. C.-C. (1982). On constructing minimum spanning trees in $k$-dimensional spaces and related problems. SIAM J. Comput. 11, 721-736. 